\title{
Nomogram to Predict Poor Outcome after Mechanical Thrombectomy at Older Age and Histological Analysis of Thrombus Composition
}

\author{
Longyan Meng, ${ }^{1}$ Haichao Wang, ${ }^{1}$ Hua Yang, ${ }^{2}$ Xiang Zhang, ${ }^{3}$ Quanbin Zhang, ${ }^{3}$ Qiong Dong, \\ Zhongwen Shu, ${ }^{3}$ Liuwei Chen, ${ }^{3}$ Li Gong $\mathbb{D}^{1},{ }^{1}$ and Yanxin Zhao ${ }^{1}{ }^{1}$ \\ ${ }^{1}$ Department of Neurology, Shanghai Tenth People's Hospital, Tongji University, Shanghai 200072, China \\ ${ }^{2}$ Department of Neurosurgery, Binhai People's Hospital, Jiangsu Province, China \\ ${ }^{3}$ Department of Neurosurgery, Shanghai Tenth People's Hospital, Tongji University, Shanghai 200072, China \\ Correspondence should be addressed to Li Gong; gongli@tongji.edu.cn and Yanxin Zhao; zhao_yanxin@126.com
}

Received 30 September 2020; Revised 9 November 2020; Accepted 30 November 2020; Published 19 December 2020

Academic Editor: Hailiang Tang

Copyright (c) 2020 Longyan Meng et al. This is an open access article distributed under the Creative Commons Attribution License, which permits unrestricted use, distribution, and reproduction in any medium, provided the original work is properly cited.

\begin{abstract}
An easy scoring system to predict the risk of poor outcome after mechanical thrombectomy among the elderly is currently not available. Therefore, we aimed to develop a nomogram for predicting the probability of negative prognosis in aged patients with acute ischemic stroke undergoing thrombectomy. In addition, we sought to investigate the association between histological thrombus composition and stroke characteristics. To this end, we prospectively studied a developed cohort using data collected from a stroke center from November 2015 to December 2019. The main outcome was functional independence, defined as a modified Rankin Scale score $\leq 2$ at 90 days following a mechanical thrombectomy. A nomogram model based on multivariate logistic models was generated. The retrieved thrombi were stained with hematoxylin and eosin and assessed according to histological composition. Our results demonstrated that age $\geq 72$ years was independently associated with poor outcome. A total of 304 participants completed the follow-up data to generate the nomogram model. After multivariate logistic regression, five variables remained independent predictors of outcome, including older age, hemorrhagic transformation, thrombolysis in cerebral infarction score, National Institute of Health Stroke score, and neutrophil-to-lymphocyte ratio, and were used to generate the nomogram. The area under the receiver-operating characteristic curve of the model was 0.803 . The clots from elderly subjects with large-artery atherosclerosis, anterior circulation, and successful recanalization groups had a higher percentage of fibrin compared to those of younger patients. This is the first nomogram to be developed and validated in a stroke center cohort for individualized prediction of poor outcome in elderly patients after mechanical thrombectomy. Clot composition provides valuable information on the underlying pathogenesis of oxidation in older patients.
\end{abstract}

\section{Introduction}

Stroke is a leading cause of serious long-term disability and mortality in China, and its incidence increases with age [1]. Advanced age is particularly related to poor poststroke functional outcomes. Mechanical thrombectomy is an effective therapy for patients with acute ischemic stroke (AIS) due to large vessel occlusions $[2,3]$. Although the latest guidelines do not recommend an upper age limit for mechanical thrombectomy, there are increasing challenges with endovascular thrombectomy in the elderly, as well as the need to consider comorbidities in clinical decision-making $[4,5]$. A high neutrophil-to-lymphocyte ratio (NLR) indicates high neutrophil counts and/or low lymphocyte counts and is considered to accurately reflect the negative effects of neutrophils, as well as the positive roles of lymphocytes in patients with stroke 
[6-8]. Our previous findings have suggested that histological thrombus composition might provide valuable information on stroke etiology [3], but its relation to outcomes after mechanical thrombectomy in older patients is less well established.

Nomograms are useful tools for clinicians to make a visualized and quick risk assessment and have been widely used for clinical decision-making in patients [9, 10]. However, nomograms have only recently been applied to predict outcomes after mechanical thrombectomy [11], and further validation is necessary among the elderly. The main objective of this study was to develop a nomogram model to predict poor outcomes after mechanical thrombectomy at an older age and to compare the diversity of histological thrombus composition.

\section{Materials and Methods}

2.1. Study Design, Participants, and Patient Consent. This was a longitudinal, prediction model development and validation study, which included data from a stroke center from November 2015 to December 2019. Participants were enrolled if they met the following criteria: age $\geq 18$ years, with anterior or posterior circulation stroke, irrespective of National Institute of Health Stroke (NIHSS) score at presentation and whether intravenous thrombolysis was performed. Patients were excluded if they were treated with intravenous thrombolysis only, missed key outcome data, or had followup loss at 3 months. The study was approved by the Ethics Committee of Shanghai Tenth People's Hospital (Shanghai, China) and was conducted in accordance with the principles of the Declaration of Helsinki. All participants and their caregivers provided written informed consent.

2.2. Outcome. The main outcome was functional independence, defined as a modified Rankin Scale (mRS) score $\leq 2$ 90 days following a mechanical thrombectomy.

2.3. Predictors and Clot Histological Analysis. Demographic and clinical data and procedural characteristics were collected at admission, as in our previous study [3]. Stroke subtypes were determined in accordance with the Trial of ORG10172 in Acute Stroke Treatment classification [12], and successful reperfusion was defined as a thrombolysis in cerebral infarction (TICI) score of 2b-3. Symptomatic intracranial hemorrhage $(\mathrm{sICH})$ was defined as any type of $\mathrm{ICH}$ with an increase of $\geq 4$ NIHSS score points from baseline within $24 \mathrm{~h}$ or leading to death [13].

As described in our previous study, all formalin-fixed, paraffin-embedded thrombi were sectioned and stained with hematoxylin-eosin [3]. The percentages of red blood cells (RBCs), white blood cells (WBCs), and fibrin in retrieved thrombi were analyzed by two experienced pathologists (LJ.F and WZ) who were blinded to the clinical data and imaging findings.

2.4. Follow-Up. A total of 322 patients enrolled in the present study were scheduled for an outpatient follow-up at 3 months. Follow-up of the mRS at 3 months was conducted by telephone or at a stroke follow-up clinic. Finally, 302 sub- jects (93.8\%) took part in the follow-up visit. The remaining 20 subjects were lost to follow-up, among whom 12 had travelled out of Shanghai and 8 refused follow-up.

2.5. Statistical Analysis. Descriptive statistics and univariate comparisons were used for statistical analysis. Comparisons were performed using Student's $t$ test or analysis of variance with Bonferroni's multiple comparisons for continuous measures, the nonparametric $t$ test or Mann-Whitney test with Dunn's multiple comparisons for noncontinuous variables, and the $\chi^{2}$ test with likelihood ratios for categorical measures. All tests were two-sided, and a $P$ value $<0.05$ was considered significant. Multivariate logistic regression analyses were used to predict good outcome and mortality (two independent models) of patients with a complete dataset. All multivariate models were assessed using the HosmerLemeshow test and $c$-statistics. Variables were included in the models on the basis of statistical significance on univariate analysis and/or clinical relevance. To generate the nomogram, multivariate logistic regression analysis was performed to evaluate the strength of the aforementioned association according to the odds ratio (OR) and corresponding 95\% confidence interval (CI) using the forward Wald method; the $F$ probability of entry was set at 0.05 and that of removal was set at 0.10 . Variables with $P$ values $<0.05$ were incorporated into the $\mathrm{R}$ language to establish the nomogram of the prediction model. The area under the receiver-operating characteristic curve (AUC-ROC) was used to calculate the predictive accuracy of the nomogram model. Then, the nomogram was subjected to bootstrapping validation $(1,000$ bootstrap resamples) to calculate a relatively corrected $C$ -index, which is equivalent to the AUC-ROC. Finally, calibration curves were plotted to assess the calibration of the nomogram by comparing the observed probability of negative outcome after endovascular thrombectomy according to the total score of the nomogram against the predicted probability based on the nomogram. A significant test statistic implied that the model was calibrated perfectly. The Hosmer-Lemeshow test was used to evaluate whether the observed event rates matched the expected rates. Statistical analyses were performed using SPSS v26 (IBM Corporation, New York, USA) and GraphPad Prism 7 (GraphPad Software, La Jolla, CA, USA).

\section{Results}

3.1. Characteristics of the Development Cohort. A total of 322 patients who met the inclusion criteria completed the followup visit at 90 days. The mean age of the patients was $69 \pm$ 11 years, and $43.4 \%$ were women. The average symptom onset-to-groin time was nearly $422 \mathrm{~min}$, and the mean NIHSS score at presentation was $14.4 \pm 4.6$. Posterior circulation stroke was treated in $18.2 \%$ of the patients. Successful reperfusion with TICI $\geq 2$ b was reported in $87.7 \%$ of subjects, and the overall rate of sICH was $31 \%$. A good outcome (mRS score, $0-2$ ) was observed in $39.1 \%$ of the cases at 90 days, and the overall mortality at 90 days was $24.4 \%$.

The ROC correlating age and probability of poor outcomes showed optimal sensitivity and specificity trade-off 
TABLE 1: Comparison of baseline characteristics and procedural and outcome measures in patients aged $<72$ years and those aged $\geq 72$ years.

\begin{tabular}{|c|c|c|c|}
\hline Baseline characteristics & $\begin{array}{l}\text { Age (years) } \\
<72(n=174)\end{array}$ & $\begin{array}{c}\text { Age (years) } \\
\geq 72(n=128)\end{array}$ & Test summary \\
\hline Sex (female) & $48(27.6 \%)$ & $83(64.8 \%)$ & 0.0001 \\
\hline Age, years (SD) & $61.94(7.51)$ & $79.50(4.59)$ & 0.0001 \\
\hline NIHSS on admission, median (range) & $13.82(4.70)$ & $15.28(4.39)$ & 0.006 \\
\hline \multicolumn{4}{|l|}{ Location } \\
\hline Anterior circulation & $136(78.2 \%)$ & $111(86.7 \%)$ & \multirow{2}{*}{0.057} \\
\hline Posterior circulation & $38(21.8 \%)$ & $17(13.3 \%)$ & \\
\hline IV tPA & $74(42.5 \%)$ & $41(32.0 \%)$ & 0.063 \\
\hline \multicolumn{4}{|l|}{ Vascular risk factors } \\
\hline Hypertension & $106(60.9 \%)$ & $90(70.3 \%)$ & 0.091 \\
\hline Diabetes mellitus & $42(24.1 \%)$ & $29(22.7 \%)$ & 0.764 \\
\hline Atrial fibrillation & $65(37.4 \%)$ & $78(60.9 \%)$ & 0.0001 \\
\hline Coronary heart disease & $17(9.8 \%)$ & $26(20.3 \%)$ & 0.010 \\
\hline \multicolumn{4}{|l|}{ Laboratory findings } \\
\hline Hemoglobin & $129.47(19.19)$ & $118.30(20.26)$ & 0.0001 \\
\hline WBC, mean (SD) & $9.03(2.96)$ & $9.41(4.41)$ & 0.366 \\
\hline Neutrophilic & $8.28(7.92)$ & $8.29(7.22)$ & 0.365 \\
\hline Lymphocyte & $1.51(3.41)$ & $1.22(1.60)$ & 0.365 \\
\hline NLR & $9.23(7.23)$ & $9.69(8.75)$ & 0.614 \\
\hline NLR $\geq 6.45$ & $98(56.3 \%)$ & $74(57.8 \%)$ & 0.796 \\
\hline C-reactive protein, mean (SD) & $20.60(37.81)$ & $21.21(33.94)$ & 0.883 \\
\hline Fibrinogen (SD) & $2.82(0.83)$ & $3.11(0.92)$ & 0.006 \\
\hline D-Dimer, mean (SD) & $3.29(7.45)$ & $3.96(5.58)$ & 0.397 \\
\hline Median onset-to-groin time, $\min (\mathrm{SD})$ & $300.85(544.50)$ & $268.95(138.11)$ & 0.518 \\
\hline $\begin{array}{l}\text { Median time from symptom onset to } \\
\text { vascular recanalization, min (SD) }\end{array}$ & $328.11(172.76)$ & $335.60(152.71)$ & 0.697 \\
\hline \multicolumn{4}{|l|}{ Outcome } \\
\hline $\begin{array}{l}\text { Reperfusion after endovascular } \\
\text { intervention TICI }(2 \mathrm{~b} / 3)\end{array}$ & $159.76(8 \%)$ & $109(85.2 \%)$ & 0.239 \\
\hline $\mathrm{ICH}$ & $57(32.8 \%)$ & $43(33.6 \%)$ & 0.879 \\
\hline Clinical outcome mRS score $(0-2)$ & $82(47.4 \%)$ & $36(28.1 \%)$ & 0.001 \\
\hline Mortality & $26(15.0 \%)$ & $36(28.1 \%)$ & 0.005 \\
\hline
\end{tabular}

NIHSS: National Institute of Health Stroke; IV tPA: intravenous recombinant tissue-type plasminogen activator; NLR: neutrophil-to-lymphocyte ratio; TICI: thrombolysis in cerebral infarction; ICH: intracerebral hemorrhage; WBC: white blood cell.

at the age of 72 years. Therefore, patients were grouped by age according to the cut-off point of 72 years, and the elderly were defined as those aged $\geq 72$ years. The average age in the elderly group was $79 \pm 5$ years compared with $61 \pm 8$ years in the younger population. Univariate analysis showed a significantly higher percentage of women in the elderly group (65\% vs. $28 \%, P<0.01$ ). Elderly patients had a significantly higher incidence of atrial fibrillation $(60.9 \%$ vs. $37.4 \%, P<$ $0.01)$. A slightly higher NIHSS score on admission was found in the elderly population compared with younger adults ( $14 \pm 5$ vs. $15 \pm 4, P=0.01$ ). There was no significant difference in the rates of posterior circulation strokes, postprocedural sICH, or treatment of intravenous thrombolysis between the two groups. For clinical outcomes, the median mRS score at 90 days was 4 in the elderly group and 3 in the younger group, and the rates of good outcomes (mRS score, $0-2)$ were $28.1 \%$ and $47.4 \%$, respectively $(P=0.0001)$ (Table 1).

3.2. Multivariate Analysis of the Development Cohort. In multivariate regression analysis, age $\geq 72$ correlated with significantly higher odds of poor outcome $(\mathrm{OR}=2.25, P=0.012)$ but was not associated with higher odds of mortality at 90 days. Additional significant predictors of poor outcomes included higher admission NIHSS scores, lower TICI scores, postprocedural sICH, diabetes, and a higher baseline NLR (Table 2).

3.3. Subgroup Analysis for Outcome Predictors. To determine if a specific clinical subgroup of subjects was associated with a 
TABLE 2: Multivariate analysis for predictors of poor outcome and mortality 90 days after endovascular thrombectomy.

\begin{tabular}{|c|c|c|c|c|c|c|}
\hline \multirow[t]{2}{*}{ Variable } & \multicolumn{3}{|c|}{$\begin{array}{l}\text { Multivariate model for poor } \\
\text { outcome }(n=302)\end{array}$} & \multicolumn{3}{|c|}{$\begin{array}{l}\text { Multivariate model for } \\
\text { mortality }(n=302)\end{array}$} \\
\hline & OR & $95 \%$ CI & $P$ value & OR & $95 \%$ CI & $P$ value \\
\hline Sex (female) & 1.54 & 0.84 to 2.82 & 0.161 & 1.33 & 0.63 to 2.79 & 0.450 \\
\hline Age $\geq 72$ years & 2.25 & 1.20 to 4.25 & 0.012 & 1.87 & 0.89 to 3.95 & 0.099 \\
\hline Onset-to-groin time & 1.00 & 0.99 to 1.01 & 0.162 & 1.00 & 0.99 to 1.00 & 0.711 \\
\hline Location & 1.54 & 0.69 to 3.48 & 0.292 & 1.32 & 0.54 to 3.20 & 0.542 \\
\hline Hypertension & 1.75 & 0.95 to 3.24 & 0.075 & 1.61 & 0.73 to 3.52 & 0.237 \\
\hline Diabetes mellitus & 2.34 & 1.16 to 4.70 & 0.017 & 3.59 & 1.69 to 7.60 & 0.001 \\
\hline Atrial fibrillation & 0.80 & 0.44 to 1.47 & 0.469 & 1.09 & 0.53 to 2.23 & 0.823 \\
\hline Coronary heart disease & 0.78 & 0.34 to 1.77 & 0.558 & 1.20 & 0.48 to 2.99 & 0.700 \\
\hline TICI score & 0.32 & 0.11 to 0.91 & 0.032 & 0.26 & 0.11 to 0.63 & 0.003 \\
\hline NIHSS score & 1.16 & 1.08 to 1.24 & 0.0001 & 1.20 & 1.10 to 1.31 & 0.0001 \\
\hline IV tPA & 0.94 & 0.51 to 1.73 & 0.839 & 1.08 & 0.52 to 2.24 & 0.844 \\
\hline $\mathrm{ICH}$ & 4.90 & 2.54 to 9.45 & 0.0001 & 2.33 & 1.17 to 4.64 & 0.016 \\
\hline NLR $\geq 6.45$ & 2.27 & 1.27 to 4.07 & 0.006 & 3.62 & 1.66 to 7.90 & 0.001 \\
\hline
\end{tabular}

NIHSS: National Institute of Health Stroke; IV tPA: intravenous recombinant tissue-type plasminogen activator; NLR: neutrophil-to-lymphocyte ratio; TICI: thrombolysis in cerebral infarction; ICH: intracerebral hemorrhage; OR: odds ratio; CI: confidence interval.

TABLE 3: Multivariate analysis for predictors of poor outcome and mortality 90 days after endovascular thrombectomy by location.

\begin{tabular}{|c|c|c|c|c|c|c|}
\hline \multirow[b]{2}{*}{ Variable } & \multicolumn{3}{|c|}{ Multivariate anterior circulation $(n=247)$} & \multicolumn{3}{|c|}{ Multivariate posterior circulation $(n=55)$} \\
\hline & OR & $95 \% \mathrm{CI}$ & $P$ value & OR & $95 \% \mathrm{CI}$ & $P$ value \\
\hline Sex (female) & 1.39 & 0.72 to 2.70 & 0.327 & 2.81 & 0.50 to 15.78 & 0.240 \\
\hline Age $\geq 72$ (years) & 2.52 & 1.25 to 5.07 & 0.010 & 0.42 & 0.06 to 3.16 & 0.398 \\
\hline Onset-to-groin time & 1.00 & 0.99 to 1.00 & 0.483 & 1.01 & 0.99 to 1.02 & 0.105 \\
\hline Hypertension & 1.81 & 0.91 to 3.60 & 0.092 & 7.53 & 0.72 to 78.43 & 0.091 \\
\hline Diabetes mellitus & 2.16 & 0.97 to 4.80 & 0.059 & 4.53 & 0.69 to 29.58 & 0.115 \\
\hline Atrial fibrillation & 0.93 & 0.48 to 1.82 & 0.834 & 0.26 & 0.05 to 1.355 & 0.110 \\
\hline NIHSS score & 1.15 & 1.06 to 1.25 & 0.001 & 1.28 & 1.05 to 1.57 & 0.016 \\
\hline IV tPA & 0.83 & 0.43 to 1.60 & 0.568 & 2.20 & 0.29 to 16.68 & 0.445 \\
\hline $\mathrm{ICH}$ & 5.40 & 2.66 to 10.96 & 0.0001 & 3.31 & 0.39 to 27.40 & 0.268 \\
\hline$N L R \geq 6.45$ & 2.15 & 1.12 to 4.12 & 0.021 & 8.80 & 1.14 to 68.00 & 0.037 \\
\hline
\end{tabular}

NIHSS: National Institute of Health Stroke; IV tPA: intravenous recombinant tissue-type plasminogen activator; NLR: neutrophil-to-lymphocyte ratio; TICI thrombolysis in cerebral infarction; ICH: intracerebral hemorrhage; OR: odds ratio; CI: confidence interval.

higher risk of poor outcome, we performed multivariate analysis to predict an mRS score $\geq 2$ at 90 days on the basis of stroke location, the extent of recanalization (TICI score), and stroke subtypes. When patients were grouped by location (anterior or posterior circulation stroke), age at presentation of $\geq 72$ was associated with lower odds of good outcomes in the anterior circulation $(\mathrm{OR}=2.52, P=0.01)$, but not in posterior circulation (Table 3 ). When stratified by stroke subtype (large-artery atherosclerosis or cardioembolism), an age at presentation of $\geq 72$ correlated with higher odds of poor outcomes in patients with cardioembolic stroke but not in those with large-artery atherosclerosis (LAA) $(\mathrm{OR}=5.42$, $P=0.002$ ) (Table 4). Similarly, when patients were grouped according to TICI scores (TICI $<2 \mathrm{~b}$ or TICI $\geq 2 \mathrm{~b}$ ), an age of $\geq 72$ years at presentation was associated with higher odds of poor outcome in patients with TICI $\geq 2 \mathrm{~b}$
$(\mathrm{OR}=2.02, \quad P=0.032) \quad($ Table 5). Additionally, when patients were grouped according to stroke location, subtype, and etiology, a high NLR showed an independent association with a higher likelihood of poor prognosis.

3.4. Development of an Individualized Prediction Model. Hypertension and diabetes did not achieve significance as variables in the binary logistic regression model and were eliminated. Five potential predictors yielded by the binomial logistic regression model (LR method) included age $\geq 72$ $(\mathrm{OR}=2.25 ; 95 \% \mathrm{CI}, 1.20-4.25)$, TICI score $(\mathrm{OR}=0.32$; 95\% CI, 0.11-0.91), NIHSS score (OR = 1.16; 95\% CI, 1.081.24), sICH $(\mathrm{OR}=4.9 ; 95 \% \mathrm{CI}, 2.54-9.45)$, and NLR $(\mathrm{OR}=2.27 ; 95 \% \mathrm{CI}, 1.27-4.07)$. A prediction model was established using the nomogram based on these five factors, and a summary of the point value of each factor used to 
TABLE 4: Multivariate analysis for predictors of poor outcome and mortality 90 days after endovascular thrombectomy by stroke subtype.

\begin{tabular}{|c|c|c|c|c|c|c|}
\hline \multirow{2}{*}{ Variable } & \multicolumn{3}{|c|}{ Multivariate model for LAA $(n=159)$} & \multicolumn{3}{|c|}{ Multivariate model for CE $(n=143)$} \\
\hline & OR & $95 \% \mathrm{CI}$ & $P$ value & OR & $95 \% \mathrm{CI}$ & $P$ value \\
\hline Sex (female) & 1.97 & 0.81 to 4.80 & 0.136 & 1.85 & 0.69 to 4.98 & 0.224 \\
\hline Age $\geq 72$ (years) & 1.04 & 0.42 to 2.57 & 0.932 & 5.42 & 1.84 to 15.95 & 0.002 \\
\hline Onset-to-groin time & 1.00 & 0.99 to 1.00 & 0.220 & 1.00 & 0.99 to 1.01 & 0.467 \\
\hline Location & 2.85 & 1.03 to 7.87 & 0.043 & 0.39 & 0.08 to 2.01 & 0.263 \\
\hline Hypertension & 2.37 & 1.00 to 5.59 & 0.050 & 1.57 & 0.55 to 4.49 & 0.400 \\
\hline Diabetes mellitus & 2.11 & 0.87 to 5.13 & 0.099 & 5.12 & 1.19 to 22.07 & 0.029 \\
\hline Coronary heart disease & 0.82 & 0.24 to 2.84 & 0.750 & 0.78 & 0.19 to 3.21 & 0.726 \\
\hline TICI score & 0.21 & 0.05 to 0.91 & 0.037 & 0.220 & 0.03 to 1.47 & 0.119 \\
\hline NIHSS score & 1.12 & 1.02 to 1.23 & 0.016 & 1.28 & 1.12 to 1.46 & 0.0001 \\
\hline IV tPA & 2.10 & 0.84 to 5.28 & 0.114 & 0.41 & 0.14 to 1.18 & 0.097 \\
\hline $\mathrm{ICH}$ & 2.73 & 1.08 to 6.92 & 0.035 & 11.100 & 3.58 to 34.38 & 0.0001 \\
\hline NLR $\geq 6.45$ & 2.34 & 1.00 to 5.44 & 0.049 & 4.53 & 1.55 to 13.23 & 0.006 \\
\hline
\end{tabular}

NIHSS: National Institute of Health Stroke; IV tPA: intravenous recombinant tissue-type plasminogenactivator; NLR: neutrophil-to-lymphocyte ratio; TICI: thrombolysis in cerebral infarction; ICH: intracerebral hemorrhage; OR: odds ratio; CI: confidence interval; LAA: large-artery atherosclerosis; CE: cardioembolism.

TABle 5: Multivariate analysis for predictors of poor outcome and mortality 90 days after endovascular thrombectomy by TICI score.

\begin{tabular}{lccc}
\hline \multirow{2}{*}{ Variable } & \multicolumn{3}{c}{ Multivariate TICI $\geq 2 \mathrm{~b}(n=265)$} \\
& OR & $95 \%$ CI & $P$ value \\
\hline Sex (female) & 1.38 & 0.74 to 2.57 & 0.31 \\
Age $\geq 72$ & 2.02 & 1.06 to 3.84 & 0.032 \\
Onset-to-groin time & 1.00 & 0.99 to 1.00 & 0.363 \\
Location & 1.49 & 0.67 to 3.33 & 0.333 \\
Hypertension & 1.71 & 0.91 to 3.20 & 0.095 \\
Diabetes mellitus & 2.55 & 1.25 to 5.22 & 0.01 \\
Atrial fibrillation & 0.86 & 0.46 to 1.61 & 0.64 \\
Coronary heart disease & 0.86 & 0.37 to 1.98 & 0.726 \\
NIHSS score & 1.15 & 1.07 to 1.23 & 0.0001 \\
IV tPA & 1.01 & 0.54 to 1.90 & 0.979 \\
ICH & 4.44 & 2.25 to 8.75 & 0.0001 \\
NLR $\geq 6.45$ & 2.29 & 1.26 to 4.15 & 0.006 \\
\hline
\end{tabular}

NIHSS: National Institute of Health Stroke; IV tPA: intravenous recombinant tissue-type plasminogen activator; NLR: neutrophil-tolymphocyte ratio; TICI: thrombolysis in cerebral infarction; ICH: intracerebral hemorrhage; OR: odds ratio; CI: confidence interval.

calculate the total score is presented in Figure 1. The AUCROC curve of the prediction model was 0.803 , with a sensitivity of $67.9 \%$ and specificity of $82.8 \%$.

3.5. Performance of the Nomogram to Predict Negative Outcomes after Endovascular Thrombectomy at Older Age. The calibration curve of the nomogram for the predicted probability of negative outcome after endovascular thrombectomy at older age in this cohort is shown in Figure 2. The results of the 1,000 bootstrap samples estimated the AUC to be 0.791 , which suggested good discrimination of the model.
3.6. Histological Analysis of Clot Composition. Of the 147 materials collected from the occlusion artery, clots were classified as cardioembolism and LAA. As shown in Figure 3(a), the fibrin composition differed significantly between the patients aged $\geq 72$ and $<72$ years $(48.7 \%$ vs. $43.8 \%, P=0.012)$. Furthermore, the clots from the elderly subjects in the LAA (42.0\% vs. $39.5 \%, P=0.002)$, anterior circulation $(58.3 .0 \%$ vs.52.6.5\%, $P=0.047)$, and successful recanalization $(44.8 \%$ vs. $41.0 \%, P=0.01$ ) groups had a higher percentage of fibrin compared with younger patients (Figures $3(\mathrm{~b})-3(\mathrm{~d})$ ).

\section{Discussion}

Our findings suggest that an older age of $\geq 72$ years is independently associated with poor outcomes despite comparable clinical characteristics and procedural variables. We present a precise nomogram model based on older age, hemorrhagic transformation, the TICI score, the NIHSS score, and NLR to predict the probability of poor outcomes following mechanical thrombectomy. This model provides clinicians with a useful tool for quick and individualized preoperative risk assessment in elderly patients aged $\geq 72$ years.

The negative prognostic effect of older age persisted despite anterior circulation, cardioembolic etiology, and successful recanalization (TICI $\geq 2 \mathrm{~b}$ ). We demonstrate that an age of $\geq 72$ years is a negative predictor of poor outcome in patients with anterior circulation occlusion but does not independently predict an increased rate of functional dependence in the posterior circulation group. Our findings and those of previous studies have consistently shown that age is an independent predictor of functional independence and mortality after thrombectomy in elderly patients with acute anterior circulation stroke $[14,15]$. Although the BASICS study demonstrated that mechanical thrombectomy is safe for acute circulation stroke, its effectiveness has not yet been confirmed [16]. There is new evidence suggesting that mechanical thrombectomy of cardioembolic large-vessel 


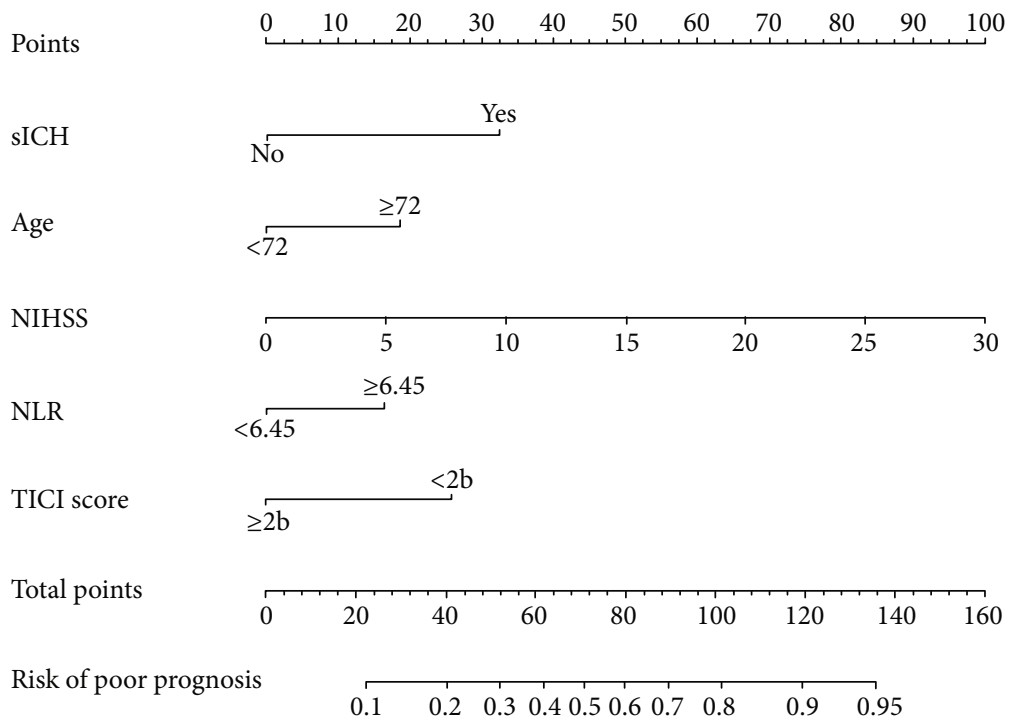

Figure 1: Nomogram for predicting the probability of poor outcome after mechanical thrombectomy. NIHSS: National Institute of Health Stroke; NLR: neutrophil-to-lymphocyte ratio; TICI: thrombolysis in cerebral infarction; ICH: intracerebral hemorrhage.

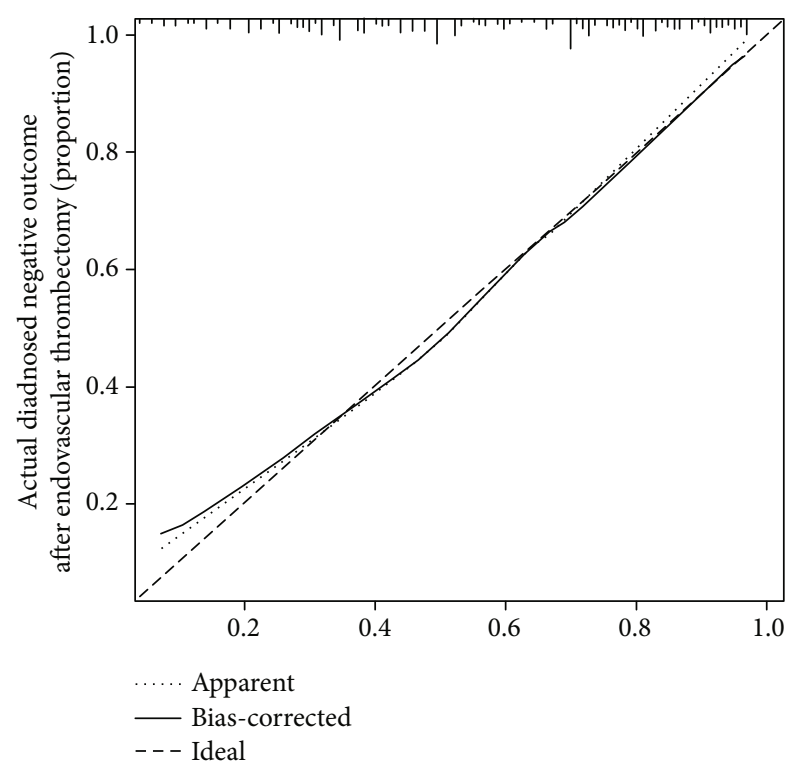

FIGURE 2: Nomogram-predicted probability of negative outcome after mechanical thrombectomy.

occlusion has a higher rate of successful reperfusion and higher efficacy compared with those of noncardioembolic etiology [17]. Our data suggest an independent association of age $\geq 72$ years with poor outcome despite cardioembolic etiology, which raised concerns about preoperative risk assessment. Consistent with existing findings, older age may predict functional dependence after mechanical thrombectomy in patients with AIS despite successful recanalization [18]. These data seem to suggest that it might be difficult for elderly patients with severe neurological impairment at baseline to undergo a successful procedure. In addition to older age, a higher NLR on admission was a strong modifiable predictor of poor outcome when patients were divided

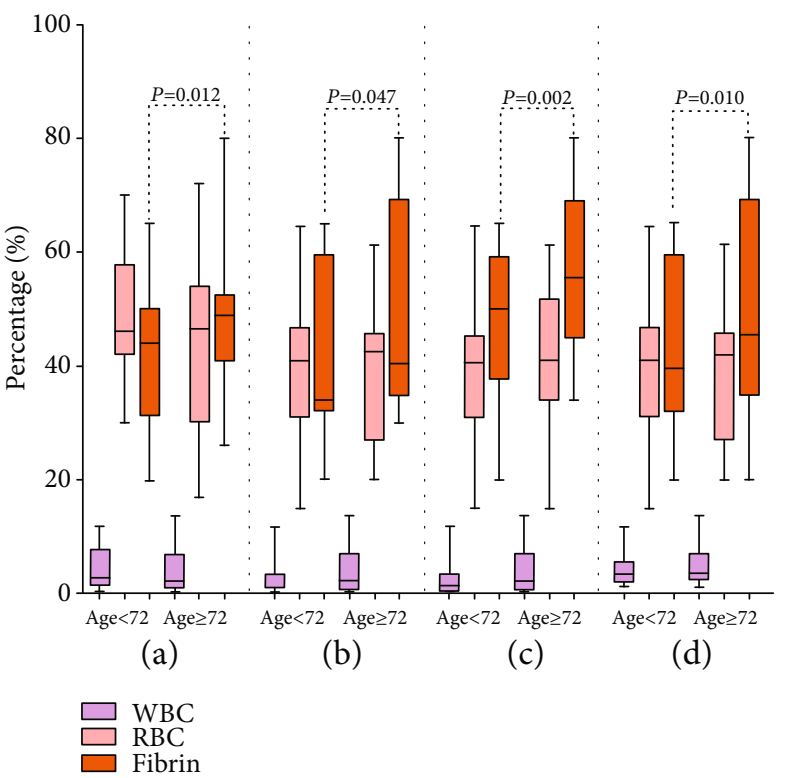

FIGURe 3: (a) Thrombi composition in patients aged $\geq 72$ and $<72$ years; (b) thrombi composition in patients aged $\geq 72$ and $<72$ years with anterior circulation stroke; (c) thrombi composition in patients aged $\geq 72$ and $<72$ years with large-artery atherosclerosis stroke; and (d) thrombi composition in patients aged $\geq 72$ and $<72$ years with successful recanalization $(\mathrm{TICI} \geq 2 \mathrm{~b})$. WBC: white blood cell; RBC: red blood cell.

according to stroke location, etiology, or extent of recanalization (TICI scores). The NLR is a potential novel biomarker of oxidative stress, which has recently been reported as an important predictor of clinical outcome after mechanical thrombectomy [19]. Our study further strengthens the importance of the NLR in predicting clinical outcomes after mechanical thrombectomy.

An important result in the present study was the establishment of a nomogram model to predict functional 
outcome and mortality, as this has not been reported previously among survivors aged $\geq 72$ years. This model showed good discrimination and calibration, indicating that the predictive power is as good as representing actual risk. The verification of the internal validation group (training group) by bootstrap analysis further confirmed the reliability of the present nomogram. Although several studies have examined the relationship between risk predictors and stroke outcome after mechanical thrombectomy $[14,15,20]$, there is growing evidence that nomograms have a better performance than risk predictors $[10,11]$. In contrast to the risk group, a nomogram model provides a visualized and individualized estimate of the prediction probability of a specific outcome for an individual patient, as well as an important tool of medical decision-making based on the individual's disease characteristics. Thus, this model, showing good predicting ability, can be used by clinicians to increase confidence in their preprocedure risk assessment.

Although our primary goal was to develop a nomogram model to predict poor outcome after mechanical thrombectomy at an older age, we also investigated differences in the histological characteristics of thrombi between the two age groups. Our results demonstrate that age at presentation $\geq 72$ years was associated with a higher proportion of fibrin in thrombi than those $<72$ years in anterior circulation, cardioembolic etiology, and successful recanalization. Increased plasma fibrin levels have been reported to be associated with a significantly higher risk of major stroke events in aged patients [21]. In the present study, we measured plasma fibrin in thrombi and found that fibrin-rich thrombi were more common in older patients. Fibrin-rich thrombi may have deleterious effects on the development of stroke by several mechanisms, including elevated friction between the clot and the vessel wall, lower successful recanalization rates, and increased number of passes at retrieval, and subsequent severe endothelial damage, all of which may lead to catastrophic outcomes and higher risk of mortality [22-24]. Moreover, oxidative stress has been reported to correlate with unfavorable changes in fibrin clot properties, including faster formation of tough thrombus and lower successful recanalization rates [25-27]. Thus, fibrin-rich thrombi may be a potential oxidative marker of older patients with large vessel occlusion undergoing mechanical thrombectomy.

The major strengths of our study include the development of a readily available and rapid tool, which can facilitate individual preprocedure identification of negative outcomes at older age, as well as homogeneous histological analysis in the included patients. However, this study has some limitations. First, this study is a single-center, observational study with a natural bias, and if possible, an external validation in a multicenter cohort with a larger patient number should be considered. Second, the relationship between functional outcome and plasma NLR may be dynamic and may be influenced by multiple variables such as diet or fever, which may have influenced the results. The dynamic NLR data will be considered in future studies because of its more effective predicting ability. Finally, the components of the retrieved thrombi might not completely reflect those of the entire thrombus, and although we attempted to exclude this unavoidable bias, this influence should be considered.

\section{Conclusion}

This study provides an easy-to-use nomogram model to predict poor outcomes after mechanical thrombectomy at an older age of $\geq 72$ years. This nomogram provides clinicians with a new tool that is more useful for the visualization of risk for each individual than traditional risk scores in the preprocedure decision-making process. In addition, we found more fibrin-rich thrombi among elderly survivors undergoing mechanical thrombectomy, suggesting that oxidative stress links older age to neurological dysfunction. Future studies are warranted to investigate this potential target in elderly stroke patients undergoing mechanical thrombectomy.

\section{Data Availability}

The data used to support the findings of this study are available from the corresponding author.

\section{Conflicts of Interest}

The authors declare that the research was conducted in the absence of any commercial or financial relationships that could be construed as a potential conflict of interest.

\section{Authors' Contributions}

LYM performed most of the experiments, interpreted the data, and wrote the first draft of the paper. HCW and YH performed part of the experiments, analyzed the data, and collected blood samples. XZ and QBZ performed most of the thrombectomy procedures. ZWS, LWC, and QD conceived the study and participated in sample collection. LG critically edited the manuscript and supervised the study. YXZ provided funding and designed the study. Longyan Meng, Haichao Wang, and HuaYang contributed equally to this work.

\section{Acknowledgments}

We are grateful to Dr. LJ Feng and Dr. W Zhang from the Department of Pathology, Shanghai Tenth People's Hospital, Tongji University, for their support with the pathology. This work was supported by grants from the National Natural Science Foundation of China (Nos. 81901183 and 81771131), the Shanghai Municipal Key Clinical Specialty (No. shslczdzk06102), and the Science and Technology Commission of Shanghai Municipality (Nos. 17411950100 and 19140900105).

\section{References}

[1] L. Gong, H. Wang, Q. Dong et al., "Intracranial atherosclerotic stenosis is related to post-stroke cognitive impairment: a crosssectional study of minor stroke," Current Alzheimer Research, vol. 17, no. 2, pp. 177-184, 2020. 
[2] M. Bladowski, J. Gawrys, D. Gajecki, E. Szahidewicz-Krupska, A. Sawicz-Bladowska, and A. Doroszko, "Role of the platelets and nitric oxide biotransformation in ischemic stroke: a translative review from bench to bedside," Oxidative Medicine and Cellular Longevity, vol. 2020, Article ID 2979260, 18 pages, 2020.

[3] L. Gong, X. Zheng, L. Feng et al., "Bridging therapy versus direct mechanical thrombectomy in patients with acute ischemic stroke due to middle cerebral artery occlusion: a clinicalhistological analysis of retrieved thrombi," Cell Transplantation, vol. 28, no. 6, pp. 684-690, 2018.

[4] A. Alawieh, R. M. Starke, A. R. Chatterjee et al., "Outcomes of endovascular thrombectomy in the elderly: a 'real-world' multicenter study," Journal of NeuroInterventional Surgery, vol. 11, no. 6, pp. 545-553, 2019.

[5] M. Martini, J. Mocco, A. Turk et al., "An international multicenter retrospective study to survey the landscape of thrombectomy in the treatment of anterior circulation acute ischemic stroke: outcomes with respect to age," Journal of NeuroInterventional Surgery, vol. 12, pp. 115-121, 2020.

[6] M. Goyal, A. M. Demchuk, B. K. Menon et al., "Randomized assessment of rapid endovascular treatment of ischemic stroke," New England Journal of Medicine, vol. 372, no. 11, pp. 1019-1030, 2015.

[7] F. Palmacci, E. Toti, A. Raguzzini et al., "Neutrophil-to-lymphocyte ratio, Mediterranean diet, and bone health in coeliac disease patients: a pilot study," Oxidative Medicine and Cellular Longevity, vol. 2019, Article ID 7384193, 14 pages, 2019.

[8] S. Lattanzi, F. Brigo, E. Trinka, C. Cagnetti, M. di Napoli, and M. Silvestrini, "Neutrophil-to-lymphocyte ratio in acute cerebral hemorrhage: a system review," Translational Stroke Research, vol. 10, no. 2, pp. 137-145, 2019.

[9] R. M. Busch, O. Hogue, M. W. Kattan et al., "Nomograms to predict naming decline after temporal lobe surgery in adults with epilepsy," Neurology, vol. 91, no. 23, pp. e2144-e2152, 2018.

[10] M. Cappellari, G. Turcato, S. Forlivesi et al., "STARTINGSICH nomogram to predict symptomatic intracerebral hemorrhage after intravenous thrombolysis for stroke," Stroke, vol. 49, no. 2, pp. 397-404, 2018.

[11] M. Cappellari, S. Mangiafico, V. Saia et al., "IER-SICH nomogram to predict symptomatic intracerebral hemorrhage after thrombectomy for stroke," Stroke, vol. 50, no. 4, pp. 909916, 2019.

[12] H. P. Adams Jr., B. H. Bendixen, L. J. Kappelle et al., “Classification of subtype of acute ischemic stroke. Definitions for use in a multicenter clinical trial. TOAST. Trial of org 10172 in acute stroke treatment," Stroke, vol. 24, no. 1, pp. 35-41, 1993.

[13] Y. Hao, D. Yang, H. Wang et al., "Predictors for symptomatic intracranial hemorrhage after endovascular treatment of acute ischemic stroke," Stroke, vol. 48, no. 5, pp. 1203-1209, 2017.

[14] M. V. Jayaraman and R. A. McTaggart, "Endovascular treatment of anterior circulation large vessel occlusion in the elderly," Frontiers in Neurology, vol. 8, 2018.

[15] Y. Ahn, S. K. Kim, B. H. Baek, Y. Y. Lee, H.-j. Lee, and W. Yoon, "Predictors of catastrophic outcome after endovascular thrombectomy in elderly patients with acute anterior circulation stroke," Korean Journal of Radiology, vol. 21, no. 1, pp. 101-107, 2020.

[16] A. Compter, BASICS Study Group, E. J. R. J. van der Hoeven et al., "Vertebral artery stenosis in the Basilar Artery Interna- tional Cooperation Study (BASICS): prevalence and outcome," Journal of Neurology, vol. 262, no. 2, pp. 410-417, 2015.

[17] S. Tiedt, M. Herzberg, C. Küpper et al., "Stroke etiology modifies the effect of endovascular treatment in acute stroke," Stroke, vol. 51, no. 3, pp. 1014-1016, 2020.

[18] Z.-S. Shi, D. S. Liebeskind, B. Xiang et al., "Predictors of functional dependence despite successful revascularization in large-vessel occlusion strokes," Stroke, vol. 45, no. 7, pp. 1977-1984, 2014.

[19] D. Lux, V. Alakbarzade, L. Bridge et al., "The association of neutrophil-lymphocyte ratio and lymphocyte-monocyte ratio with 3-month clinical outcome after mechanical thrombectomy following stroke," Journal of Neuroinflammation, vol. 17, no. 1, p. 60, 2020

[20] A. Malhotra, X. Wu, S. Payabvash et al., "Comparative effectiveness of endovascular thrombectomy in elderly stroke patients," Stroke, vol. 50, no. 4, pp. 963-969, 2019.

[21] S. G. Wannamethee, P. H. Whincup, L. Lennon, A. Rumley, and G. D. Lowe, "Fibrin D-dimer, tissue-type plasminogen activator, von Willebrand factor, and risk of incident stroke in older men," Stroke, vol. 43, no. 5, pp. 1206-1211, 2012.

[22] E. V. Griemert, K. Recarte Pelz, K. Engelhard, M. K. Schäfer, and S. C. Thal, "PAI-1 but not PAI-2 gene deficiency attenuates ischemic brain injury after experimental stroke," Translational Stroke Research, vol. 10, no. 4, pp. 372-380, 2019.

[23] S. Duffy, R. McCarthy, M. Farrell et al., "Per-pass analysis of thrombus composition in patients with acute ischemic stroke undergoing mechanical thrombectomy," Stroke, vol. 50, no. 5, pp. 1156-1163, 2019.

[24] M.-S. Sun, H. Jin, X. Sun et al., "Free radical damage in ischemia-reperfusion injury: an obstacle in acute ischemic stroke after revascularization therapy," Oxidative Medicine and Cellular Longevity, vol. 2018, Article ID 3804979, 17 pages, 2018.

[25] C. C. Helms, S. Kapadia, A. C. Gilmore, Z. Lu, S. Basu, and D. B. Kim-Shapiro, "Exposure of fibrinogen and thrombin to nitric oxide donor ProliNONOate affects fibrin clot properties," Blood Coagulation \& Fibrinolysis, vol. 28, no. 5, pp. 356-364, 2017.

[26] V. S. Fennell, S. V. Setlur Nagesh, K. M. Meess et al., "What to do about fibrin rich 'tough clots'? Comparing the Solitaire stent retriever with a novel geometric clot extractor in an in vitro stroke model," Journal of NeuroInterventional Surgery, vol. 10, no. 9, pp. 907-910, 2018.

[27] J. P. Bembenek, M. Niewada, J. Siudut, K. Plens, A. Członkowska, and A. Undas, "Fibrin clot characteristics in acute ischaemic stroke patients treated with thrombolysis: the impact on clinical outcome," Thrombosis and haemostasis, vol. 117, no. 7, pp. 1440-1447, 2017. 